\title{
Performance Degradation Modeling for Multi-State Element Considering Aging Factor
}

\author{
Zhiqiang LI*, Tingxue XU**, Junyuan GU***, Linyu FU****, Jianzhong ZHAO***** \\ *Naval Aeronautical University, Yantai 264001, China,E-mail: 18663813941@163.com \\ **Naval Aeronautical University, Yantai 264001,China,E-mail: yt-xtx@163.com \\ ***Naval Aeronautical University, Yantai 264001, China,E-mail: 763607429@qq.com \\ ****Naval Aeronautical University, Yantai 264001, China,E-mail: 2249949260@qq.com \\ *****Naval Aeronautical University, Yantai 264001, China, E-mail: honeyfox_ytlzq@hotmail.com \\ cross'ref http://dx.doi.org/10.5755/j01.mech.24.2.19183
}

\section{Nomenclature and assumptions}

\section{Nomenclature}

MSE - Multi-state element; MSS - Multi-state system; BN-Bayesian network; DBN-Dynamic Bayesian network; ARA-Arithmetic Reduction of Age; ARI-Arithmetic Reduction of Intensity; UGF - Universal generating function; $k$-Perfect state for MSE; $\lambda_{i, j}$-Failure rate transiting from state $i$ to state $j ; \quad \Lambda$-Instantaneous state-transition rate matrix; $p_{i}(t)$-Probability function of MSE at state $i$ at constant $t ; g_{i}$-Performance level of MSE; $u(z, t)-u$-function of MSE at constant $t ; U_{s}(z, t)-u$-function of MSS at constant $t$; $p_{s k_{i}}(t)$-Probability function of MSS at state $k_{i}$ at constant $t ; \quad g_{s k_{i}}$-Performance level of MSS; $w$ - Constant demand of MSS; $R(t)-$ Reliability of MSS at instant $t$.

\section{Assumptions}

1. During the useful life period of an element, its time lingering at a state follows exponential distribution;

2. During the wear-out life period of an element, its failure rate increase with time;

3. In the Markov process, the state probability at a future instant, given the present state of the process, does not rely on the states occupied in the past;

4. A MSS is put into use at $t=0$ in perfect functionality and maintenance measures are taken when its reliability drops below the given value; or in parallel;

5. MSS is combined with multiple MSEs in series

6. The performance level of MSS can be detected immediately according to its electric parameters or output performance.

\section{Introduction}

Multi-state elements (MSEs) refer to those that have different performance levels, including perfect functionality, complete failure and intermediate states [1-3]. Systems composed of MSEs and having multiple performance levels determined by these elements are called multi-state systems (MSSs). MSS theory draws a lot of attention nowadays and a variety of researches are carried out around this topic.

Markov process is widely used to describe statetransition between different states of MSE and MSS [2, 4-
6]. Anatoly et al. [2] presented a multi-state Markov model for a power generating unit to calculate important reliability indices. In literature [6], Anatoly et al. put forward several methods to model for non-repairable elements and repairable elements by applying Markov process. To obtain the reliability and availability of complex systems, dynamic Bayesian network (DBN) and universal generating function (UGF) are adopted in many researches.

DBN, developed on Bayesian network $(\mathrm{BN})$ and hidden Markov process, has advantages in reasoning forward and backward and predicting the change of random variables. Liu et al. [7, 8] established a dynamic Bayesian network (DBN) of subsea blowout preventer to perform reliability analysis and common cause failure analysis. Daniele et al.[9] established a DBN framework inside a system or among systems to assess cascading effects for a power grid. However, it is difficult to obtain conditional probability values for DBN because of lacking of data. In this case, UGF which expresses discrete random variables using algebraic procedures is a better choice. The reliability of MSS under desired performance level is calculated by using polynomial assemble operator through like-terms collection calculation. Mi et al. [10] performed reliability analysis of an excavator rectifier feedback system by applying modified Markov and belief UGF approach. Li and Enrico [11] put forward an analytical multi-state modeling approach to assess the reliability of electric power systems on the base of UGF.

During the useful life period of equipment, its failure rate is approximately regarded as a constant. For a MSE, state-transition rates between states are also regarded as constants. In the process of modeling, state-transition rates and repair time are all constants $[12,13]$. With the increasing of service years, the equipment moves from the useful life period toward the wear-out life period. And a phenomenon occurs that failures happen more frequently than before. By referring to recorded data, it's obvious that the failure rate and state-transition rates between states are not constants any more. Models based on virtual age and imperfect repair are proposed to offer suggestions in making maintenance decisions [14-16]. Maria et al. [17] put forward models based on Arithmetic Reduction of Age (ARA) and Arithmetic Reduction of Intensity (ARI) to analyze imperfect repair. Fan et al. [18] made a cost effective strategy to minimize the total cost of imperfect degradation-based maintenance under imperfect repair.

However, these aforementioned models focus on binary state elements and systems which are not available 
for MSEs and MSSs anymore. For example, visual life models by applying Kijima I and Kijima II are only suitable for binary reliability models. Aiming at this limitation, this paper will establish a degradation model for MSE considering aging factor under period maintenance. During the useful life period continuous time Markov models are established for MSEs, while non-homogenous continuous time Markov models will be built during the wear-out life period by introducing an aging factor. In order to obtain the reliability of MSSs, UGF is adopted to combine the performance levels and probability functions of MSEs through combination operators. By comparing the reliability during the useful life period with that during the wear-out life period, maintenance measure can be adjusted to maintain a desired reliability value.

\section{Stochastic degradation for MSE during the useful life period}

During the useful life period of MSE, degradation begins from the perfect state $k$ to lower state $i(1 \leq i<k)$. Markov process can be applied to model this state-transition process which assumes that the next state of the element only relates to its current state and the transition time follows exponential distribution. State-transition rate of MSE is a constant denoted as $\lambda_{i, j}(i>j, i 、 j \in[1,2, \cdots, k])$. The stochastic degradation process is shown in Fig. 1, and its instantaneous rate matrix $\Lambda$ is denoted as follow:

$$
\Lambda=\left[\begin{array}{ccccc}
\lambda_{k, k} & \lambda_{k, k-1} & \cdots & \lambda_{k, 2} & \lambda_{k, 1} \\
0 & \lambda_{k-1, k-1} & \cdots & \lambda_{k-1,2} & \lambda_{k-1,1} \\
\vdots & \vdots & \ddots & \vdots & \vdots \\
0 & 0 & \cdots & \lambda_{2,2} & \lambda_{2,1} \\
0 & 0 & \cdots & 0 & \lambda_{1,1}
\end{array}\right] .
$$

According to the stochastic degradation process, differential equations can be written to obtain state probabilities for the Markov process presented in Fig. 1:

$$
\left\{\begin{array}{l}
\frac{d p_{k}(t)}{d t}=-p_{k}(t) \sum_{e=1}^{k-1} \lambda_{k, e} \\
\frac{d p_{i}(t)}{d t}=\sum_{e=i+1}^{k} \lambda_{e, i} p_{e}(t)-p_{i}(t) \sum_{e=1}^{i-1} \lambda_{i, e}, i=2,3, \cdots, k-1, \\
\frac{d p_{1}(t)}{d t}=\sum_{e=2}^{k} \lambda_{e, 1} p_{e}(t),
\end{array}\right.
$$

where $p_{i}(t)=\operatorname{Pr}\{X(t)=i\}$ is the state probability of $X(t)$.

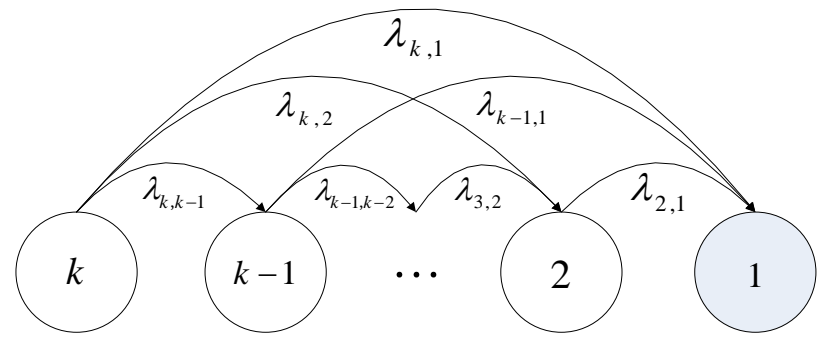

Fig. 1 Stochastic degradation process for MSE during its useful life period

Because the degradation begins from the perfect state $k$, the initial conditions can be denoted as:

$$
p_{k}(0)=1, p_{k-1}(0)=p_{k-2}(0)=\cdots=p_{1}(0)=0 .
$$

Through Laplace-Stieltjes transformation, state functions of MSE in different states can be obtained:

At instant $t$, the probability that the MSE is in state $k$ is:

$$
\begin{aligned}
p(\Phi(t)=k) & =\exp \left[-\left(\lambda_{k, k-1}+\lambda_{k, k-2}+\cdots+\lambda_{k, 1}\right) t\right]= \\
& =\exp \left[-\left(\sum_{i=1}^{k-1} \lambda_{k, i}\right) t\right] .
\end{aligned}
$$

At instant $t$, the probability that the MSE is in state

$$
p(\Phi(t)=k-1)=\int_{0}^{t} \exp \left[-G_{k} \tau_{1}\right] \lambda_{k, k-1} \exp \left[-G_{k-1}\left(t-\tau_{1}\right)\right] d \tau_{1}=\frac{\lambda_{k, k-1}}{G_{k}-G_{k-1}}\left\{\exp \left(-G_{k-1} t\right)-\exp \left(-G_{k} t\right)\right\},
$$

where: $G_{i}=\sum_{j=1}^{i-1} \lambda_{i, j}$. To obtain $p(\Phi(t)=k-2)$, the MSE can get to state $k-2$ from state $k$ through state $k-1$,

$$
\begin{aligned}
p_{1}(\Phi(t)=k-2) & =\int_{0}^{t} \int_{0}^{\tau_{2}} \exp \left[-G_{k} \tau_{1}\right] \lambda_{k, k-1} \exp \left[-G_{k-1}\left(\tau_{2}-\tau_{1}\right)\right] \lambda_{k-1, k-2} \exp \left[-G_{k-2}\left(t-\tau_{2}\right)\right] d \tau_{1} d \tau_{2}= \\
& =\frac{\lambda_{k, k-1} \lambda_{k-1, k-2}}{G_{k}-G_{k-1}}\left\{\frac{\exp \left(-G_{k-2} t\right)-\exp \left(-G_{k-1} t\right)}{G_{k-1}-G_{k-2}}-\frac{\exp \left(-G_{k-2} t\right)-\exp \left(-G_{k} t\right)}{G_{k}-G_{k-2}}\right\} .
\end{aligned}
$$

Or the MSE get to state $k-2$ from state $k$ directly:

$$
p_{2}(\Phi(t)=k-2)=\int_{0}^{t} \exp \left[-G_{k} \tau_{2}\right] \lambda_{k, k-2} \exp \left[-G_{k-2}\left(t-\tau_{2}\right)\right] d \tau_{2}=\frac{\lambda_{k, k-2}}{G_{k}-G_{k-2}}\left\{\exp \left(-G_{k-2} t\right)-\exp \left(-G_{k} t\right)\right\} .
$$

For MSE, there are two ways to degrade to state $k-2$ from state $k$, four ways to degrade to state $k-3$ from state $k$, eight ways to degrade to state $k-4$ from state $k$. There are $\sum_{j=0}^{k-i-1}\left(\begin{array}{c}k-i-1 \\ j\end{array}\right)$ ways to degrade to 
state $i$ from state $k$. Without loss of generality, the probability function that the MSE is in state $k$ at instant $t$ can be summarized as follows:

1. Degrade from state $k$ to state $i$ directly (without intermediate states):

$$
\begin{aligned}
p_{0}(t) & =\int_{0}^{t} \exp \left[-G_{k} \tau_{1}\right] \lambda_{k, i} \exp \left[-G_{i}\left(t-\tau_{1}\right)\right] d \tau_{1}= \\
& =\frac{\lambda_{k, i}}{G_{k}-G_{i}}\left\{\exp \left(-G_{i} t\right)-\exp \left(-G_{k} t\right)\right\} .
\end{aligned}
$$

2. Degrade from state $k$ to state $i$ with one intermediate state $j$ :

$$
p_{1, j}(t)=\lambda_{k, j} \lambda_{j, i} \int_{0}^{t} \int_{0}^{\tau_{2}} \exp \left[-G_{k} \tau_{1}\right] \exp \left[-G_{k}\left(\tau_{2}-\tau_{1}\right)\right] \exp \left[-G_{i}\left(t-\tau_{2}\right)\right] d \tau_{1} d \tau_{2},
$$

where $j=[i+1, \cdots, k-1]$.

3. Degrade from state $k$ to state $i$ with $n(n=3,4, \cdots, k-i-1)$ intermediate states:

$$
p_{n}(t)=\lambda_{k, j_{1}}\left(\prod_{l=1}^{n-1} \lambda_{j_{l}, j_{l+1}}\right) \lambda_{j_{n}, i} \int_{0}^{t} \cdots \int_{0}^{\tau_{2}} \exp \left[-G_{k} \tau_{1}\right]\left(\prod_{m=1}^{n} \exp \left[-G_{j_{m}}\left(\tau_{m+1}-\tau_{m}\right)\right]\right) \exp \left[-G_{i}\left(t-\tau_{n+1}\right)\right] d \tau_{1} \cdots d \tau_{n+1},
$$

where $j_{1}=[k-1, k-2, \cdots, i+n], 1<j_{n}<\cdots<j_{1}<k$. For any $n$, there are $\left(\begin{array}{c}k-i-1 \\ r\end{array}\right)$ combinations.

\section{Aging degradation for MSE during the wear-out life period}

During the wear-out life period of the "bath curve", the degradation of MSE is not only related to its current state, but also depends on its service age. Weibull distribution is widely used to model degradation process for binary state equipment, which is not suitable for multi-state equipment $[18,19]$. Non-homogenous continuous time Markov model (NHCTMM) is applied here to model the degradation of aging MSE [20, 21], and the corresponding process is shown in Fig. 2.

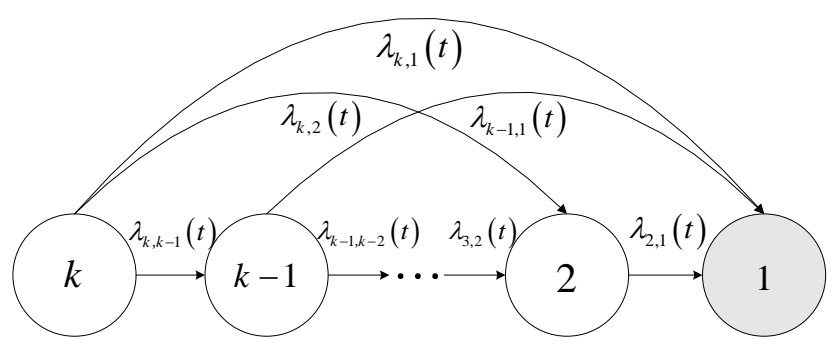

Fig. 2 Aging degradation process for MSE during wear-out life period

Let $\Phi(t) \in[1,2, \cdots, k]$ represent the state of MSE at instant $t$. The transition probability from instant $s$ to

$$
p(\Phi(t)=k-1)=\int_{0}^{t} \exp \left[-\int_{0}^{\tau_{1}} \sum_{j=1}^{k-1} \lambda_{k, j}(s) d s\right] \lambda_{k, k-1}\left(\tau_{1}\right) \exp \left[-\int_{\tau_{1}}^{t} \sum_{j=1}^{k-2} \lambda_{k-1, j}(s) d s\right] d \tau_{1} .
$$

Similarly, to obtain $p(\Phi(t)=k-2)$, the MSE can get to state $k-2$ from state $k$ through state $k-1$,

$$
p_{1}(\Phi(t)=k-2)=\int_{0}^{t} \exp \left[-\int_{0}^{\tau_{2}} \sum_{j=1}^{k-1} \lambda_{k, j}(s) d s\right] \lambda_{k, k-2}\left(\tau_{2}\right) \exp \left[-\int_{\tau_{2}}^{t} \sum_{j=1}^{k-3} \lambda_{k-2, j}(s) d s\right] d \tau_{2} .
$$

Or the MSE get to state $k-2$ from state $k$ directly. instant $t$ to transit state $i$ to state $j$ can be denoted as:

$$
p_{i, j}(s, t)=\operatorname{Pr}(\Phi(t)=j \mid \Phi(s)=i) \text {, }
$$

where $s<t, i, j \in[1,2, \cdots, k], j<i$.

Similar to Markov process, the instantaneous statetransition matrix $\Lambda(t)$ for NHCTMM can be denoted as:

$\Lambda(t)=\left[\begin{array}{ccccc}\lambda_{k, k}(t) & \lambda_{k, k-1}(t) & \cdots & \lambda_{k, 2}(t) & \lambda_{k, 1}(t) \\ 0 & \lambda_{k-1, k-1}(t) & \cdots & \lambda_{k-1,2}(t) & \lambda_{k-1,1}(t) \\ \vdots & \vdots & \ddots & \vdots & \vdots \\ 0 & 0 & \cdots & \lambda_{2,2}(t) & \lambda_{2,1}(t) \\ 0 & 0 & \cdots & 0 & \lambda_{1,1}(t)\end{array}\right]$,

where $\lambda_{i, j}(t)=\lim _{\Delta t \rightarrow \infty} \frac{\operatorname{Pr}(\Phi(t+\Delta t))-\operatorname{Pr}(\Phi(t))}{\Delta t}$.

Combined with the initial conditions in Eq. (3), NHCTMP equation can be solved to determine the probability functions in different states of MSE:

At instant $t$, the probability that the MSE is in state $k$ is:

$$
p(\Phi(t)=k)=\exp \left[-\int_{0}^{t} \sum_{j=1}^{k-1} \lambda_{k, j}(t) d \tau\right]
$$

At instant $t$, the probability that the MSE is in state $k-1$ is: 


$$
\begin{aligned}
& p_{2}(\Phi(t)=k-2)=\int_{0}^{t} \int_{0}^{\tau_{2}} \exp \left[-\int_{0}^{\tau_{1}} \sum_{j=1}^{k-1} \lambda_{k, j}(s) d s\right] \lambda_{k, k-1}\left(\tau_{1}\right) \exp \left[-\int_{\tau_{1}}^{\tau_{2}} \sum_{j=1}^{k-2} \lambda_{k-1, j}(s) d s\right] \lambda_{k-1, k-2}\left(\tau_{2}\right) \\
& \exp \left[-\int_{\tau_{2}}^{t} \sum_{j=1}^{k-3} \lambda_{k-2, j}(s) d s\right] d \tau_{1} d \tau_{2} .
\end{aligned}
$$

The probability function that the MSE is in state $k$ at instant $t$ can be summarized as follows:
1. Degrade from state $k$ to state $i$ directly (without intermediate states):

$$
p_{0, i}(t)=\int_{0}^{t} \exp \left[-\int_{0}^{\tau_{1}} \sum_{j=1}^{k-1} \lambda_{k, j}(s) d s\right] \lambda_{k, i}\left(\tau_{1}\right) \exp \left[-\int_{\tau_{1}}^{t} \sum_{j=1}^{i-1} \lambda_{i, j}(s) d s\right] d \tau_{1} .
$$

2. Degrade from state $k$ to state $i$ with one intermediate state $l \in[i+1, i+2, \cdots, k-1]$ :

$$
p_{1, l, i}(t)=\int_{0}^{t} \int_{0}^{\tau_{2}} \exp \left[-\int_{0}^{\tau_{1}} \sum_{j=1}^{k-1} \lambda_{k, j}(s) d s\right] \lambda_{k, l}\left(\tau_{1}\right) \exp \left[-\int_{\tau_{1}}^{\tau_{2}} \sum_{j=1}^{l-1} \lambda_{l, j}(s) d s\right] \lambda_{l, i}\left(\tau_{2}\right) \exp \left[-\int_{\tau_{2}}^{t} \sum_{j=1}^{i-1} \lambda_{i, j}(s) d s\right] d \tau_{1} d \tau_{2} .
$$

3. Degrade from state $k$ to state $i$ with $n(n=3,4, \cdots, k-i-1)$ intermediate states:

$$
\begin{aligned}
& p_{(n, i), h}(t)=\int_{0}^{t} \cdots \int_{0}^{\tau_{2}} \exp \left[-\int_{0}^{\tau_{1}} \sum_{j=1}^{k-1} \lambda_{k, j}(s) d s\right] \lambda_{k, l_{1}}\left(\tau_{1}\right) \prod_{g=1}^{n-1}\left\{\exp \left[-\int_{\tau_{N}}^{\tau_{g+1}} \sum_{j=1}^{l_{g-1}} \lambda_{l_{g}, j}(s) d s\right] \lambda_{l_{N}, l_{g+1}}\left(\tau_{g+1}\right)\right\} \\
& \exp \left[-\int_{\tau_{n}}^{\tau_{i}} \sum_{j=1}^{l_{n-1}} \lambda_{l_{n}, j}(s) d s\right] \lambda_{l_{n}, l_{i}}\left(\tau_{g+1}\right) \exp \left[-\int_{\tau_{i}}^{t} \sum_{j=1}^{i-1} \lambda_{i, j}(s) d s\right] d \tau_{1} \cdots d \tau_{n+1},
\end{aligned}
$$

where $h=\left(\begin{array}{c}k-i-1 \\ r\end{array}\right), l_{g}=[i+1, i+2, \cdots, k-1]$,

$i<l_{n}<\cdots<l_{1}<k, g=1,2, \cdots, n$.

\section{Degradation modeling for MSS}

Universal Generating Function (UGF) is widely used in reliability evaluation and risk assessment for MSS [22-24]. Performance levels and probability functions of MSE are expressed in the form of polynomial represented by discrete variables. The UGF of MSS can be obtained through combination operators determined by logic relations between MSEs.

UGF of a MSE can be denoted as:

$$
\begin{aligned}
& u(z, t)=\sum_{i=1}^{k} p_{i}(t) z^{g_{i}}=p_{1}(t) z^{g_{1}}+p_{2}(t) z^{g_{2}}+ \\
& +\cdots+p_{k}(t) z^{g_{k}},
\end{aligned}
$$

where $g_{i} \in\left[g_{1}, g_{2}, \cdots, g_{k}\right]$ is a performance level of MSE, and $p_{i}(t)$ is its corresponding probability function.

UGF of MSS can be denoted as:

$$
\begin{aligned}
& U_{s}(z, t)=\sum_{i=1}^{k_{s}} p_{s i}(t) z^{g_{s i}}=p_{s 1}(t) z^{g_{s 1}}+p_{s 2}(t) z^{g_{s 2}}+ \\
& +\cdots+p_{s k_{s}}(t) z^{g_{s k_{s}}},
\end{aligned}
$$

where $g_{s k_{i}} \in\left[g_{s k_{1}}, g_{s k_{2}}, \cdots g_{s k_{s}}\right]$ is a performance level of
MSS, and $p_{s k_{i}}(t)$ is its corresponding probability function.

Usually, MSS can be simplified as a series-parallel structure. In order to determine the performance parameters of MSS, UGF of elements in the MSS are obtained firstly. Then, the parallel system regarded as a subsystem is replaced by an equivalent element in series. Keep doing this until there is no parallel subsystem in the system. At last, UGF of MSS is determined by the remaining series system. A MSS model with $N$ parallel subsystems is shown in Fig. 3.

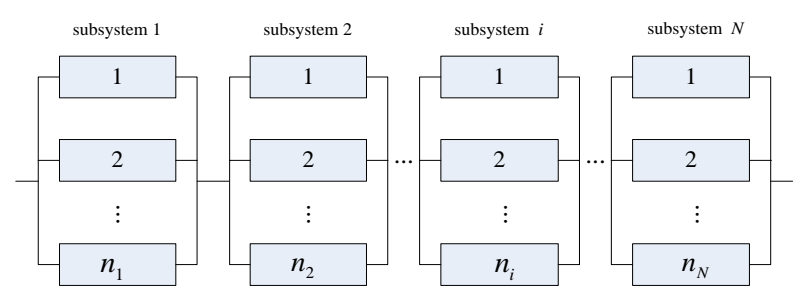

Fig. 3 A MSS model with $N$ parallel subsystems

For subsystem i with $n_{i}$ elements in parallel, the states' performance between the subsystem and its elements can be denoted as:

$$
X_{i}=f\left(G_{i 1}, G_{i 2}, \cdots, G_{i n_{i}}\right),
$$

where $X_{i}$ represents the performance of subsystem $i$; $G_{i 1}, G_{i 2}, \cdots, G_{i i_{i}}$ represent the performance of elements in the parallel system.

UGF of subsystem $i$ can be denoted as: 


$$
\begin{aligned}
& U_{i}(z, t)=\Omega\left(U_{i 1}(z, t), U_{i 2}(z, t), \cdots, U_{i n_{i}}(z, t)\right)= \\
& =\sum_{k=1}^{M_{i}} p_{i k}(t) z^{x_{i k}(t)},
\end{aligned}
$$

where $M_{i}$ is the state number of subsystem $i$; $\left\{x_{i 1}(t), x_{i 2}(t), \cdots, x_{i M_{i}}(t)\right\}$ represents the state performance of subsystem $i$ at instant $t$;

$\left\{p_{i 1}(t), p_{i 2}(t), \cdots, p_{i M_{i}}(t)\right\}$ represents its corresponding state probability.

Assume that a MSS is consisted of $N$ subsystems in series, then the states' performance between the MSS and its subsystem can be denoted as:

$$
Y=f\left(X_{1}, X_{2}, \cdots, X_{N}\right),
$$

where $Y$ represents the performance of MSS;

$X_{1}, X_{2}, \cdots, X_{N}$ represent the performance of subsystems.

UGF of MSS can be denoted as:

$$
\begin{aligned}
& U(z, t)=\Omega\left(U_{1}(z, t), U_{2}(z, t), \cdots, U_{N}(z, t)\right)= \\
& =\sum_{s=1}^{M_{s y s}} p_{s}(t) z^{y_{s}(t)},
\end{aligned}
$$

where $M_{s y s}$ is the state number of MSS;

$\left\{y_{1}(t), \cdots, y_{M_{s y s}}(t)\right\}$ represents the state performance of

MSS at instant $t ;\left\{p_{1}(t), p_{2}(t), \cdots, p_{M_{s y s}}(t)\right\}$ represents its corresponding state probability.

Use the following operator $\delta$ to obtain the system availability at instant $t$ for any demand $w$ :

$$
\begin{aligned}
& \delta(U(z, t), w)=\delta\left(\sum_{s=1}^{M_{s y s}} p_{s}(t) z^{y_{s}(t)}, w\right)= \\
& =\sum_{s=1}^{M_{s y s}} \delta\left(p_{s}(t) z^{y_{s}(t)}, w\right),
\end{aligned}
$$

where $\delta\left(p_{s}(t) z^{y_{s}(t)}, w\right)=\left\{\begin{array}{l}p_{s}(t), y_{s}(t) \geq w \\ 0, y_{s}(t)<w\end{array} . w \quad\right.$ represents the constant demand of MSS. When the state performance of MSS is higher than $w$, the system is available. The reliability of MSS at instant $t$ can be denoted as:

$$
R(t)=\delta(U(z, t), w)=\sum_{y_{s}(t) \geq w} p_{s}(t) .
$$

\section{Case study}

A compressor system of an engine is consisted of three aging elements, a first-stage rotor represented by element 1 , a second-stage rotor represented by element 2 and a stator represented by element 3, shown in Fig. 4 . The firststage rotor and the second-stage rotor play important roles in air suction and compression. The first-stage rotor has two states, having $40 \%$ and 0 performance level for state 2 and state 1 respectively. The second-stage rotor has three states, having $60 \%, 30 \%$ and 0 performance level for state 3 , state 2 and state 1 respectively. The stator offering assistance for the rotors has four states, having $100 \%, 60 \%, 30 \%$ and 0 performance level for state 4 , state 3 , state 2 and state 1 respectively. The efficiency of the compressor is determined by these two rotors and the stator. According to recorded data and consulting domain experts, state-transition rates for these three elements during the useful life period and the wear-out life period are listed in Table 1.

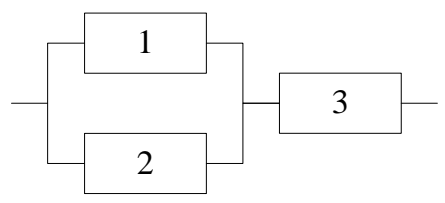

Fig. 4 Structure of the compressor system

Table 1

State-transition rates for elements in the compressor

\begin{tabular}{|c|c|c|c|c|c|c|}
\hline \multirow{2}{*}{$\begin{array}{c}\text { Ele- } \\
\text { ment }\end{array}$} & \multicolumn{3}{|c|}{$\begin{array}{c}\text { Rate during the } \\
\text { useful life period } \\
(/ y)\end{array}$} & \multicolumn{3}{|c|}{ Rate during the wear-out life } \\
& $\lambda_{2,1}$ & $\lambda_{3,2}$ & $\lambda_{4,3}$ & $\lambda_{2,1}(t)$ & $\lambda_{3,2}(t)$ & $\lambda_{4,3}(t)$ \\
\hline 1 & 0.2 & - & - & $0.2+0.1 t^{2}$ & - & - \\
\hline 2 & 0.6 & 0.3 & - & $0.6+0.2 t$ & $0.3+0.1 t$ & - \\
\hline 3 & 0.8 & 0.4 & 0.2 & $0.8+0.4 t$ & $0.4+0.2 t$ & $0.2+0.1 t$ \\
\hline
\end{tabular}

\subsection{Degradation modeling for MSE}

To perform degradation analysis, element 3 with four states is taken for example. According to Eqs. (8)-(10), probability functions in different states for element 3 during the useful life period can be obtained as follow:

$$
\left\{\begin{array}{l}
p(\Phi(t)=4)=e^{-1.4 t}, \\
p(\Phi(t)=3)=-e^{-1.4 t}+e^{-1.2 t}, \\
p(\Phi(t)=2)=\frac{2}{3} e^{-1.4 t}-e^{-1.2 t}+\frac{1}{3} e^{-0.8 t} \\
p(\Phi(t)=1)=-\frac{2}{3} e^{-1.4 t}-\frac{1}{3} e^{-0.8 t}+1
\end{array}\right.
$$

Similarly, according to Eqs. (17)-(19), probability functions in different states for element 3 during the wearout life period can be obtained as follow:

$$
\left\{\begin{array}{l}
p(\Phi(t)=4)=e^{-0.35 t^{2}-1.4 t} \\
p(\Phi(t)=3)=-e^{-0.35 t^{2}-1.4 t}+e^{-0.3 t^{2}-1.2 t} \\
p(\Phi(t)=2)=\frac{2}{3} e^{-0.35 t^{2}-1.4 t}+\frac{1}{3} e^{-0.2 t^{2}-0.8 t}-e^{-0.3 t^{2}-1.2 t} \\
p(\Phi(t)=1)=-\frac{2}{3} e^{-0.35 t^{2}-1.4 t}-\frac{1}{3} e^{-0.2 t^{2}-0.8 t}+1
\end{array}\right.
$$

Probability curves of element 3 in different states during the useful life period and the wear-out life period are depicted in Fig. 5. Where $P 3-i(i \in[4,3,2,1])$ represents the probability curve of element 3 during the useful life period, and $P 3-i^{\prime}\left(i^{\prime} \in[4,3,2,1]\right)$ represents the probability curve of element 3 during the wear-out life period. Because the existence of aging factor, the probability curve in state 4 
of element 3 during the wear-out life period drops more rapidly to 0.0150 than that to 0.0608 during the useful life period. As two intermediate states, there are transitions from upper state to state 3 and state 2 and transitions from these states to state 1 . Probabilities in state 3 and state 2 are relatively lower all the time. However, the probability curve in state 1 increases rapidly with the increasing of time. And it's obvious that because of aging factor the probability in state 1 during the wear-out life period is higher arriving at 0.9598 than that arriving at 0.8922 during the useful life period, which is consistent with the practice.

In order to obtain the reliability curve and failure curve of element 3 , state 4 , state 3 and state 2 are summed as an operation state, shown in Fig. 6. Influenced by aging

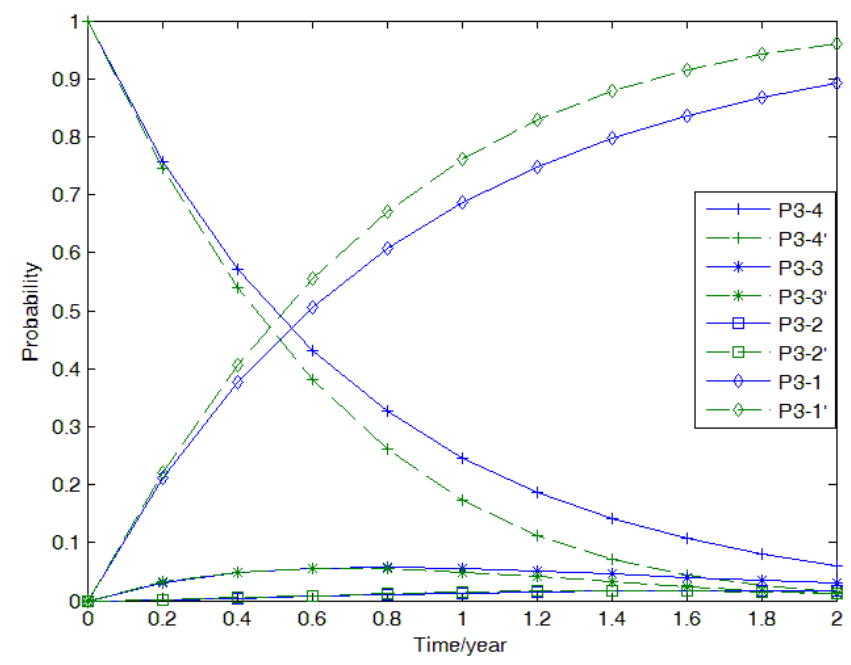

Fig. 5 Probability curves of element 3 in four states factor, the reliability of element 3 is lower than that without aging factor. At the end of one year, the reliability of element 3 during the useful life period will reach at 0.3142 , while that during the wear-out life period will reach at 0.2385 .

By referring to literature $[13,20]$, the same result can be obtained to verify our model during the useful life period without considering aging factor.

\subsection{Reliability analysis for MSS}

According to Eqs. (8)-(10) and Eqs. (17)-(19), probability functions for element 1 and element 2 in different states are listed in Table 2.

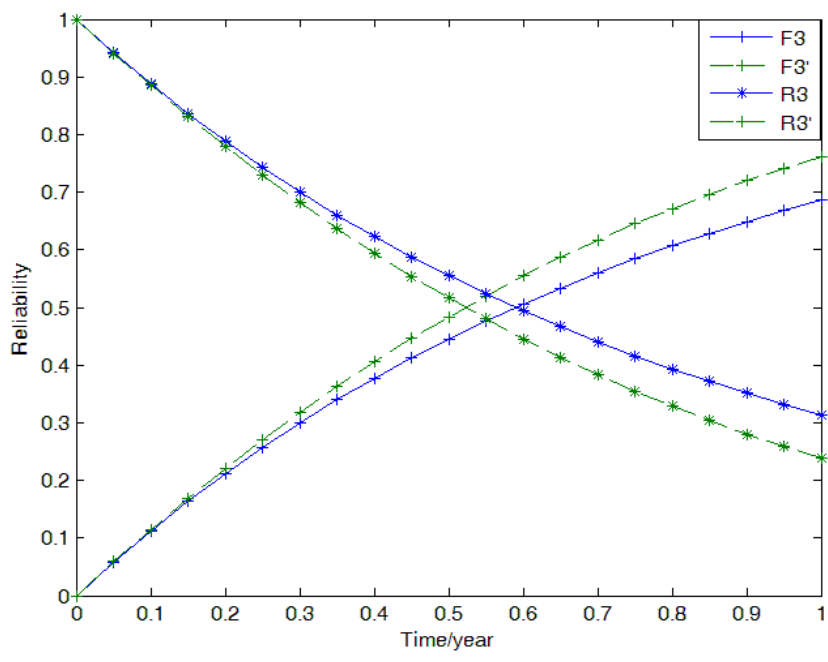

Fig. 6 Reliability curve of element 3

Table 2

State probability functions for element 1 and element 2

\begin{tabular}{|c|c|c|c|c|c|c|}
\hline \multirow{2}{*}{ Element } & \multicolumn{2}{|c|}{ During the useful life period } & \multicolumn{3}{|c|}{ During the wear-out life period } \\
\cline { 2 - 7 } & 1 & 2 & 3 & 1 & 2 & 3 \\
\hline 1 & $1-e^{-0.2 t}$ & $e^{-0.2 t}$ & - & $1-e^{-\frac{1}{30} t^{3}-\frac{1}{5} t}$ & $e^{-\frac{1}{30} t^{3}-\frac{1}{5} t}$ & - \\
\hline 2 & $1-e^{-0.6 t}$ & $e^{-0.6 t}-e^{-0.9 t}$ & $e^{-0.9 t}$ & $1-e^{-0.1 t^{2}-0.6 t}$ & $e^{-0.1 t^{2}-0.6 t}-e^{-0.15 t^{2}-0.9 t}$ & $e^{-0.15 t^{2}-0.9 t}$ \\
\hline
\end{tabular}

UGF for three elements can be denoted as:

$$
\begin{aligned}
& u_{1}(z, t)=p_{(1,1)}(t) \cdot z^{g_{(1,1)}}+p_{(1,2)}(t) \cdot z^{g_{(1,2)}}, \\
& u_{2}(z, t)=p_{(2,1)}(t) \cdot z^{g_{(2,1)}}+p_{(2,2)}(t) \cdot z^{g_{(2,2)}}+ \\
& +p_{(2,3)}(t) \cdot z^{g_{(2,3)}}, \\
& u_{3}(z, t)=p_{(3,1)}(t) \cdot z^{g_{(3,1)}}+p_{(3,2)}(t) \cdot z^{g_{(3,2)}}+ \\
& +p_{(3,3)}(t) \cdot z^{g_{(3,3)}}+p_{(3,4)}(t) \cdot z^{g_{(3,4)}} .
\end{aligned}
$$

Because element 1 and element 2 are connected in a parallel subsystem, a new element can be used to replace the subsystem. Then the performance of the whole system can be determined by the new element and element 3 . UGF of the compressor is written as follow and its probability function in different states are listed in Table 3.

$$
\begin{aligned}
& U_{s}(z, t)=\otimes\left(\otimes\left(u_{1}(z, t), u_{2}(z, t)\right), u_{3}(z, t)\right)= \\
& =\otimes\left(\otimes\left(\sum_{i_{1}=1}^{2} p_{\left(1, i_{1}\right)} z^{g_{\left(1, i_{1}\right)}}, \sum_{i_{2}=1}^{3} p_{\left(2, i_{2}\right)} z^{\left.g_{\left(2, i_{2}\right)}\right)}\right), \sum_{i_{3}=1}^{4} p_{\left(3, i_{3}\right)} z^{g_{\left(3, i_{3}\right)}}\right)= \\
& =\sum_{i_{3}=1}^{4} \sum_{i_{2}=1}^{3} \sum_{i_{1}=1}^{2}\left(\prod_{j=1}^{3} p_{\left(j, i_{j}\right)}(t) z^{\min \left(g_{\left(1, i_{1}\right)}+g_{\left(2, i_{2}\right)}, g_{\left(3, i_{3}\right)}\right)}\right) .
\end{aligned}
$$

Reliability curves of the compressor under different demand values are drawn in Fig. 7. If $w>70 \%$, the reliability during the useful life period will arrive at 0.7844 while that during the wear-out life period will arrive at 0.7815 at the end of $0.2 y$. If $0<w \leq 30 \%$, the reliability during the useful life period will arrive at 0.6065 while that during the wear-out life period will arrive at 0.5944 at the end of $0.2 y$. It's obvious that the higher the demand is, more quickly the curve will drop. In order to ensure its operation time and avoid unnecessary dropdown, reasonable demand should be set. Comparing to the curves during the useful life period, the curves during the wear-out life period have relative lower reliability values. Thus, when making 
maintenance measures, aging factors should be taken into consideration.

Table 3

Performance levels and probability functions of the compressor

\begin{tabular}{|c|c|}
\hline Performance & Probability function \\
\hline$g_{s 1}=0 \%$ & $p_{(1,1)}(t) p_{(2,1)}(t)+p_{(3,1)}(t)\left[p_{(1,1)}(t) p_{(2,2)}(t)+p_{(1,1)}(t) p_{(2,3)}(t)+p_{(1,2)}(t)\right]$ \\
\hline$g_{s 2}=30 \%$ & $p_{(1,1)}(t) p_{(2,2)}(t)\left[p_{(3,2)}(t)+p_{(3,3)}(t)+p_{(3,4)}(t)\right]+p_{(3,2)}(t)\left[p_{(1,1)}(t) p_{(2,3)}(t)+p_{(1,2)}(t)\right]$ \\
\hline$g_{s 3}=40 \%$ & $p_{(1,2)}(t) p_{(2,1)}(t)\left[p_{(3,3)}(t)+p_{(3,4)}(t)\right]$ \\
\hline$g_{s 4}=60 \%$ & $p_{(1,1)}(t) p_{(2,3)}(t)\left[p_{(3,3)}(t)+p_{(3,4)}(t)\right]+p_{(3,3)}(t)\left[p_{(1,2)}(t) p_{(2,2)}(t)+p_{(1,2)}(t) p_{(2,3)}(t)\right]$ \\
\hline$g_{s 5}=70 \%$ & $p_{(1,2)}(t) p_{(2,2)}(t) p_{(3,4)}(t)$ \\
\hline$g_{s 6}=100 \%$ & $p_{(1,2)}(t) p_{(2,3)}(t) p_{(3,4)}(t)$ \\
\hline
\end{tabular}

Assume the demand is $50 \%$, reliability function for the compressor can be denoted as:

$$
\begin{aligned}
& R(t)=\sum_{i=1}^{6} p_{s i}(t) \cdot 1\left(\left(y_{s i}-50 \%\right) \geq 0\right)==p_{(1,1)}(t) p_{(2,3)}(t)\left[p_{(3,3)}(t)+p_{(3,4)}(t)\right]+p_{(3,3)}(t)\left[p_{(1,2)}(t) p_{(2,2)}(t)+p_{(1,2)}(t) p_{(2,3)}(t)\right]+ \\
& +p_{(1,2)}(t) p_{(2,2)}(t) p_{(3,4)}(t)+p_{(1,2)}(t) p_{(2,3)}(t) p_{(3,4)}(t) .
\end{aligned}
$$

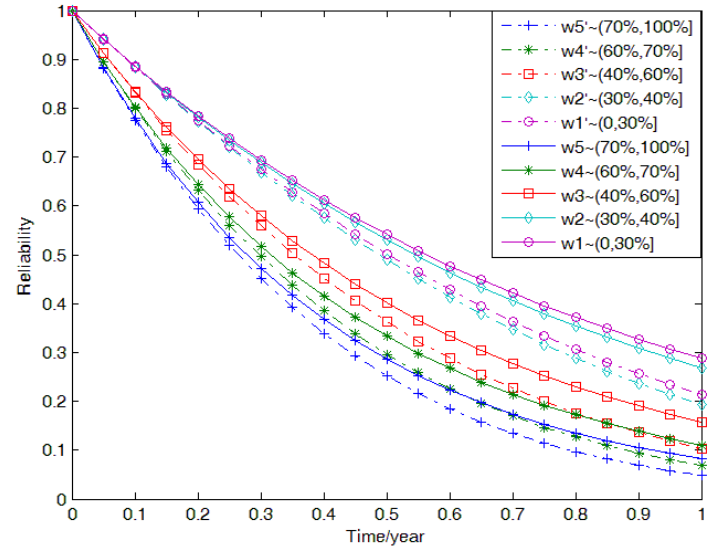

Fig. 7 Reliability curves under different demand values

The corresponding reliability curves are $w 3 \in(40 \%, 60 \%]$ and $w 3^{\prime} \in(40 \%, 60 \%]$ shown in Fig. 7 . To maintain a reliability level at $80 \%$, maintenance circle during the useful life period is forty-five days, while it's forty-three days during the wear-out life period when taking aging factor into consideration.

\section{Conclusions}

On the base of state-transition modeling for MSE during the useful life period, model for degradation process is established during the wear-out life period by introducing aging factor.

1. For MSE in the useful life period, state-transition process can be determined by setting continuous time Markov model.

2. When aging factor is considered, state-transition rates between states for MSE are no longer constants. By solving non-homogenous continuous time Markov model, state probability functions can be obtained.

3 . To calculate the reliability of MSS under different demand values, UGF of MSS should be solved on the base of UGFs for elements and the combination operator.
It's obvious that the reliability is relatively lower when considering aging factor, which can offer suggestions in making maintenance measures.

\section{Acknowledgements}

This work is supported by Natural Science Foundation of China (No. 51605487), Natural Science Foundation of Shandong Province (ZR2016FQ03), China Postdoctoral Science Foundation (2016M592965). All support is gratefully acknowledged.

\section{References}

1. Wang, L. Q.; Zhou, W. J.; Wei, X. S.; Zhai, L. L.; Wu, G K. 2016. A coupling vibration model of multi-stage pump rotor system based on FEM, Mechanika 22: 31-37. http://dx.doi.org/10.5755/j01.mech.22. 1. 11420.

2. Anatoly, L.; David, E.; David, L.; Hanoch, B. H. 2012. A multi-state Markov model for a short-term reliability analysis of a power generating unit, Reliability Engineering and System Safety 98: 1-6.

http://dx.doi.org/10. 1016/j. ress. 2011.10. 008.

3. Zhou, W. J.; Wei, X. S; Zhai, L. L; Wei, X. S; Wang, L. Q. 2014. Nonlinear characteristics and stability optimization of rotor-seal-bearing system, Journal of Vibroengineering 16(2): 818-831. http://www.jve.lt/Vibro/JVE-2014-16-2/JVE0161403. 1196.html.

4. Ninna, R. J. 2016. Scenario-based life insurance prognoses in a multi-state Markov model, Europine Actuarial Journal 6: 307-330. http://dx.doi.org/10. 1007/s13385-016-0137-8.

5. Malcolm, J. P.; Nicky, J. W.; Ades, A. E. 2011. Parameterization of treatment effects for meta-analysis in multi-state Markov models, Statistics in Medicine 30: 140-151. http://dx.doi.org/10. 1002/sim.4059.

6. Anatoly, L.; Ilia, F.; Yi, D. 2010. Multi-state System Reliability Analysis and Optimization for Engineers and Industrial Managers, Springer London Dordrecht Heidelberg New York. 
7. Cai, B. P.; Liu, Y. H.; Zhang, Y. W.; Fan, Q.; Yu, S. L. 2013. Dynamic Bayesian networks based performance evaluation of subsea blowout preventers in presence of imperfect repair, Expert Systems with Applications 40: 7544-7554. http://dx.doi. org/10.1016/j. eswa. 2013.07.064.

8. Liu, Z. K.; Liu, Y. H.; Cai, B. P.; Zhang, D. W.; Zheng, C. 2015. Dynamic Bayesian network modeling of reliability of subsea blowout preventer stack in presence of common cause failures, Journal of Loss Prevention in the Process Industries 38: 58-66.

http://dx.doi.org/10.1016/j.jlp.2015. 09. 001.

9. Daniele, C. R.; Andrea, B.; Stefania, M.; Luigi, P. 2012. A dynamic Bayesian network based framework to evaluate cascading effects in a power grid, Engineering Applications of Artificial Intelligence 25: 683-697. http://dx.doi.org/10. 1016/j. engappai. 2010.06.005.

10. Mi, J. H.; Li, Y.F.; Huang, H. Z.; Li, A. F.; Wang, X. M. 2015. Reliability analysis of excavator rectifier feedback system with multi-state components based on belief universal generating function method, Journal of Shanghai Jiaotong University (Science) 20: 344-348. http://dx.doi.org/10. 1007/s12204-015-1634-7.

11. Li, Y. F; Enrico, Z. 2012. A multi-state model for the reliability assessment of a distributed generation system via universal generating function, Reliability Engineering and System Safety 106: 28-36.

http://dx.doi.org/10. 1016/j. ress. 2012. 04.008.

12.Li, Z. Q.; Gu, J. Y.; Xu, T. X.; Fu, L. Y.; Jin, A.; Dong, Q. 2017. Reliability analysis of complex system based on dynamic fault tree and dynamic Bayesian network, Proceeding of 2nd International Conference on Reliability Systems Engineering. http://dx.doi.org/10.1109/ICRSE. 2017. 8030783.

13. Li, Z. Q.; Xu, T. X.; Gu, J. Y.; An, J.; Dong, Q. 2017. Availability modeling and analyzing of multi-state control unit under condition-based maintenance, Acta ArmamentarII 38: 2240-2250. http://dx.doi.org/10.3969/j. issn. 1000-1093. 2017. 11. 022.

14. Monika, T.; Rajiv, N. R.; Nomesh, B. 2014. Imperfect repair modeling using Kijima type generalized renewal process, Reliability Engineering and System Safety 124: 24-31.

http://dx.doi.org/10. 1016/ j. ress. 2013. 10. 007.

15. Dinh, T. N.; Yann, D.; Mitra, F. 2017. Analytical properties of an imperfect repair model and application in preventive maintenance scheduling, European Journal of Operational Research 256: 439-453. http://dx.doi.org/10. 1016/j.ejor.2016.06.026.

16. Maxim, F. 2015. On the optimal degree of imperfect repair, Reliability Engineering and System Safety 138: 54-58.

http://dx.doi.org/10. 1016/ j. ress. 2015. 01. 010.

17. Maria, L. G. T.; Marta, A. F.; Enrico, A. C.; Gustavo, L. G. 2015. ARA and ARI imperfect repair models: Estimation, goodness-of-fit and reliability prediction, Reliability Engineering and System Safety, 140: 107115.

http://dx.doi.org/10.1016/j. ress. 2015. 03. 035.

18. Fan, W.; Seyed, A. N.; John E. K. 2015. A cost effective degradation-based maintenance strategy under imperfect repair, Reliability Engineering and System
Safety 144: 234-243.

http://dx.doi.org/10. 1016/j. ress. 2015. 08.002.

19. Zhao, X. J.; Xie, M. 2017. Using accelerated life tests data to predict warranty cost under imperfect repair, Computers \& Industrial Engineering 107: 223-234. http://dx.doi.org/10. 1016/ j. cie. 2017. 03. 021.

20. Liu, Y.; Huang, H. Z. 2010. Optimal replacement policy for multi-state system under imperfect maintenance, IEEE Transactions on Reliability 3: 483-495. http://dx.doi.org/10. 1109/TR. 2010. 2051242.

21. Krishna, B. M. 2008. Handbook of performability engineeri, Springer-Verlag London Limited.

22. Jin, T. C.; Zhou, M.; Li, G. Y. 2017. Universal generating function based probabilistic production simulation for wind power integrated power systems, Journal of Modern Power Systems and Clean Energy 5: 134-141. http://dx.doi.org/10. 1007/s40565-015-0114-2.

23. Bentolhoda, J.; Lance, F. 2016. A universal generating function-based multi-state system performance model subject to correlated failures, Reliability Engineering and System Safety 152: 16-27.

http://dx.doi.org/10. 1016/j. ress. 2016.02. 004.

24. Meena, K. S.; Vasanthi, T. 2016. Reliability analysis of mobile ad hoc networks using universal generating function, Quality and Reliability Engineering International 32: 111-122.

http://dx.doi.org/10. 1002/qre. 1731.

\section{Z.Q. Li, T.X. Xu, J.Y. Gu, L.Y. Fu, J.Z. Zhao}

\section{PERFORMANE DEGRADATION MODELING FOR MULTI-STATE ELEMENT CONDERING AGING FACTOR}

S u m m a r y

With the increasing of service years, failure happens more frequently than before, which is consistent with the failure rate of equipment during the wear-out life period. On the base of continuous time Markov model for multistate elements during the useful life period, aging factor is introduced to establish a non-homogenous continuous time Markov model during the wear-out life period. To obtain the reliability of a multi-state element, universal generating function is applied to combine its performance levels and probability functions. Through a combination operator determined by series-parallel logic relations, universal generating function of a multi-state system can be obtained. At last, a compressor of an engine is taken for example. The reliability of the system and its elements considering aging factor are relatively lower than that during the useful life period. And to maintain a reliability level at $80 \%$ under the demand of $50 \%$, two days will be reduced from the planned maintenance cycle.

Keywords: Markov model; aging factor; multi-state system; universal generating function.

Received October 19, 2017

Accepted April 18, 2018 